\title{
MicroRNA-196a promotes cell proliferation and inhibits apoptosis in human ovarian cancer by directly targeting DDX3 and regulating the PTEN/ PI3K/AKT signaling pathway
}

\author{
JIE NI ${ }^{*}$, LI CHEN* ${ }^{*}$, LI LING, MENGFEI WU, QIONGZHEN REN and WEIPEI ZHU \\ Department of Obstetrics and Gynecology, The Second Affiliated Hospital of Soochow University, \\ Suzhou, Jiangsu 215004, P.R. China
}

Received January 9, 2020; Accepted May 12, 2020

DOI: $10.3892 / \mathrm{mmr} .2020 .11236$

\begin{abstract}
MicroRNAs (miRNAs/miRs) are small noncoding RNAs 19-24 nucleotides in length, which can play an important role in tumor development. However, the influence of miRNAs on the tumorigenicity of ovarian cancer cells has not been fully elucidated. Previously, DEAD box protein (DDX) 1 has been shown to play a role in tumor suppression of ovarian cancer progression. However, the functions of other DDX members in ovarian cancer development remain largely unknown. In the present study, it was demonstrated that overexpression of miR-196a promoted ovarian cancer cell proliferation. In addition, DDX3 was significantly downregulated in ovarian cancer cell lines relative to the normal ovarian cell line. Moreover, DDX3 was identified as a direct target of miR-196a in ovarian cancer cells. In addition, a preliminary mechanistic investigation indicated that downregulation of DDX3 promoted ovarian cancer cell proliferation through the PTEN/PI3K/AKT pathway. Taken together, this confirmed an association between DDX3 and miR-196a in ovarian cancer, and showed that miR-196a promoted the proliferation of ovarian cancer cells and attenuated apoptosis by downregulating the expression of DDX3 through the PTEN/PI3K/AKT pathway. Overall, the results of the present study suggested that DDX3 could be a novel target for ovarian cancer treatment.
\end{abstract}

Correspondence to: Professor Weipei Zhu, Department of Obstetrics and Gynecology, The Second Affiliated Hospital of Soochow University, 1055 Sanxiang Road, Suzhou, Jiangsu 215004, P.R. China

E-mail: weipeizhu211@126.com

*Contributed equally

Key words: miR-196a, DDX3, PTEN/PI3K/AKT pathway, ovarian cancer

\section{Introduction}

Ovarian cancer is one of the most common types of gynecologic cancer, resulting in the mortality of hundreds of thousands of women every year worldwide. Ovarian cancer is the seventh most common cause of cancer-associated mortality in women (1). Emerging evidence has supported the incessant ovulation theory as the mechanism underlying ovarian cancer; this theory suggests that recurrent ovulation-induced repair of the ovarian surface epithelium raises the likelihood of DNA damage and carcinogenesis, and thereby increases the risk of developing ovarian cancer $(2,3)$. Over the past decade, optimal resection, chemotherapy and combination therapy have evolved as ovarian cancer treatments to substantially improve patient outcomes and 5-year survival rates (4). Nevertheless, the overall patient outcome is far from ideal; thus, there remains a need for revolutionary new treatments of ovarian cancer. One strategy for identifying novel treatment targets and developing treatments involves elucidating the regulatory molecular mechanisms and key signaling pathways that underlie tumor growth and metastasis in ovarian cancer.

MicroRNAs (miRNAs/miRs) are small noncoding endogenous RNAs that mediate expression of their target gene via genetic transcription or mRNA cleavage (5). Although the precise regulatory mechanisms of miRNAs have not been fully elucidated, they are known to function in various biological processes, including cell proliferation, differentiation, morphogenesis and apoptosis (6). Previously, the abnormal expression of miRNAs has been associated with carcinogenesis and tumor development in human cancers (7-9). Thus, miRNAs are speculated to function as oncogenes or tumor suppressors, depending on their functional targets; consequently, they have great therapeutic potential as cancer treatment targets.

Previous studies have indicated that miR-196a plays a critical role in cell proliferation and tumor progression. For example, overexpression of miR-196a has been shown to accelerate cell differentiation of osteoporosis in a mouse model through regulation of the GNAS-Hedgehog signaling pathway (10). In addition, Ren et al (11), demonstrated that inhibition of miR-196a attenuated cell proliferation and glycolysis, but elicited apoptosis, in hepatocellular carcinoma 
by targeting suppressor of cytokine signaling 2 . Furthermore, high levels of miR-196a expression have been found to be related to poor survival in patients with ovarian cancer; thus, it is a potential biomarker for the clinical prognosis of ovarian carcinoma $(12,13)$. A preliminarily evaluation of the regulatory mechanisms of miR-196a in ovarian cancer suggested that miR-196a may act as an oncogene to promote migration and invasion through the downstream target gene homeobox A10 (HOXA10) (14). Despite these findings, the regulatory mechanisms of miR-196a in ovarian cancer are yet to be fully elucidated.

DEAD box protein RNA helicase 3 (DDX3) belongs to the DDX protein family, which is highly conserved in all eukaryotes. DDX3 has attracted increasing attention in cancer research due to its critical function in cancer progression. For example, the downregulation of DDX3 was shown to increase cell metastasis in colorectal cancer (15), whereas the activation of DDX3 promoted cell proliferation in breast epithelial cells (16); thus, the role of DDX3 in cancer progression is complex. In preliminary bioinformatics analysis, the target prediction software DIANA was used to determine the potential targets of miR-196a: DDX3 was found to be a direct target of miR-196a. Therefore, the relationship between miR-196a and DDX3 may be important in the regulation of ovarian cancer development.

In the present study, this relationship was further investigated. It was revealed that upregulation of miR-196a in ovarian cancer promoted cell proliferation but inhibited apoptosis. It was also demonstrated that miR-196a may have an important regulatory role in ovarian cancer progression by targeting DDX3 through regulation of the PTEN/PI3K/AKT signaling pathway. Together, these findings suggested that miR-196a and DDX3 might be potential targets for future treatments of ovarian cancer.

\section{Materials and methods}

Clinical samples and cell lines. Ovarian cancer samples and matched normal tissue samples were collected from 30 patients (median age 50, range 30-69 years) with ovarian cancer who were treated at The Second Affiliated Hospital of Soochow University (Suzhou, China) between January 2018 and July 2019. The inclusion criteria was as follows: Age 18-80; newly diagnosed with epithelial carcinoma of the ovary, which was histologically or pathologically confirmed; no prednisone use within 14 days of sampling; no previous chemotherapy or radiotherapy; no concurrent diseases. The patients had not received chemoradiotherapy before samples were collected and all ovarian cancer tissue samples were dissected from resected tumors. Written informed consent was obtained from all patients, and the present study was approved by the Institutional Ethics Committee and Academic Committee of The Second Affiliated Hospital of Soochow University.

The ovarian cancer cell lines OVCAR3, CAOV-3, A2780 and ES-2, along with the immortalized ovarian epithelial cell line HS832, were obtained from ATCC. Cells were cultured in DMEM (Gibco; Thermo Fisher Scientific, Inc.) containing $10 \%$ fetal calf serum (HyClone; GE Healthcare Life Sciences) at $37^{\circ} \mathrm{C}$ and $5 \% \mathrm{CO}_{2}$. In order to elucidate the regulatory mechanisms of the interaction between miR-196a and DDX3, OVCAR3 ovarian cancer cells were treated with LY294002 (10 $\mu \mathrm{M}$; cat. no. 19-142; Sigma-Aldrich; Merck KGaA) or DMSO for $6 \mathrm{~h}$, and then the following methods were used to determine cell proliferation and apoptosis in OVCAR3 ovarian cancer cells treated with LY294002.

Cell transfection. miR-196a mimic (5'-GCTCTGGCT CCGTGTCTTCACTCCC-3'), miR-negative control (NC) mimic (5'-UUCUCCGAACGUGUCACGUTT-3'), miR-196a inhibitor (5'-GGGAGTGAAGACACGGAGCCAGAGC-3') and miR-NC inhibitor (5'-CAGUACUUUUGUGUA GUACAA-3') were provided by Shanghai Sangon Biotech Corporation. The miRNAs $(50 \mathrm{nM})$ were mixed with Lipofectamine ${ }^{\circledR} 2000$ reagent (GE Healthcare Life Sciences) and were incubated for $15 \mathrm{~min}$ at room temperature. Subsequently, the sequences were transfected into OVCAR 3 and CAOV-3 cells plated at $1 \times 10^{5}$ cells/well in 6 -well plates. Cells were collected for analysis $48 \mathrm{~h}$ after transfection.

Reverse transcription-quantitative PCR (RT-qPCR). Total RNA from tissues and cell lines was extracted with TRIzol ${ }^{\circledR}$ reagent (Invitrogen; Thermo Fisher Scientific, Inc.), according to the manufacturer's protocol, and RNAs were reverse transcribed into cDNA at $42^{\circ} \mathrm{C}$ for $1 \mathrm{~h}$, then $90^{\circ} \mathrm{C}$ for $5 \mathrm{~min}$, using a TaqMan microRNA RT kit (Invitrogen; Thermo Fisher Scientific, Inc.). To determine the relative expression levels of targeted genes, RT-qPCR was performed using a SYBR-Green PCR kit (Invitrogen; Thermo Fisher Scientific, Inc.) on a real-time PCR system (Bio-Rad Laboratories, Inc.). The primer sequences were as follows: miR-196a, forward (F) 5'-GCTCTGGCTCCGTGTCTTCACTCCC-3', reverse (R) 5'-TGCCCCAGCACAGCCCCCGTCCCTC-3'; DDX3, F 5'-CGGCAGCATCAAATGTTTCAG-3', R 5'-AAC TGGCAGGTAGAAGGCAACTC-3'; GAPDH, F 5'-AATGGGCAGCCGTTAGGAAA-3', R 5' TGAAGG GGTCATTGATGGCA-3'; and U6, F 5'-CTCGCTTCG GCAGCACATATA-3' and R 5' ACGCTTCACGAATTT GAGTGTC-3'. The PCR thermocycling conditions were as follows: Initial denaturation at $95^{\circ} \mathrm{C}$ for $2 \mathrm{~min}$, and 40 cycles of $95^{\circ} \mathrm{C}$ for $10 \mathrm{sec}$ and $60^{\circ} \mathrm{C}$ for $1 \mathrm{~min}$. miR-196a and DDX3 mRNA levels were quantified using the $2^{-\Delta \Delta \mathrm{Cq}}$ method and normalized to the internal reference gene U6 or GAPDH, respectively (17).

Plasmid and short hairpin RNA (shRNA) transfection. OVCAR3 and CAOV-3 cells were plated into a 6-well plate (1x10 5 cells/well), cultured overnight, and were then transfected with $50 \mathrm{nM}$ corresponding vectors using Lipofectamine ${ }^{\circledR} 2000$ (Invitrogen; Thermo Fisher Scientific, Inc.), according to the manufacturer's instructions. The shRNA targeting DDX3 (shDDX3), control shRNA (shCtrl), pcDNA-DDX3 and the control plasmid pcDNA3.1 were provided by Novogene Co., Ltd. The sequences of the targeted genes were as follows: shDDX3, F 5'-AACCCACCACAGCUAGAACTT-3', R 5'-AAG UUCUAGCUGUGGUGGGTT-3'; shCtrl, F 5' UUCUCC GAACGUGUCACGUTT-3'，R 5' ACGUGACACGUU CGGAGAATT-3'; and pcDNA-DDX3, F 5' GAAGCT ACTAGAGGTTTCTAC-3' and R 5' TCTCAACATCAC 
TGAAACTTTC-3'. Analyses were performed $48 \mathrm{~h}$ after transfection and replicated three times to produce the experimental data.

MTT assays. OVCAR3 and CAOV-3 cells were added to a 96-well dish at $1 \times 10^{3}$ cells/well and were then transfected with $50 \mathrm{nM}$ miR-196 mimic, miR-NC mimic, miR-196 inhibitor, miR-NC inhibitor, pcDNA-DDX3, pcDNA-3.1, shDDX3 or shCtrl. Subsequently, $10 \mu 1$ MTT solution was added to each well at $0,24,48,72$ and $96 \mathrm{~h}$. The supernatant was discarded after $4 \mathrm{~h}$ of incubation at $37^{\circ} \mathrm{C}$ and then $100 \mu \mathrm{l}$ DMSO was added to each well to dissolve the formazan. The absorbance at $570 \mathrm{~nm}$ was determined using an enzyme-labeling instrument (EL808; BioTek Instruments, Inc.).

Cell proliferation assay. Cell proliferation assays were performed using a cell proliferation colorimetric ELISA-BrdU kit (Roche Diagnostics), according to the manufacturer's protocol. Briefly, OVCAR3 and CAOV-3 cells were cultured in a 96-well plate at a density of $1 \times 10^{3}$ cells/well. Overnight, the cells were transfected with $50 \mathrm{nM}$ miR-196 mimic, miR-NC mimic, miR-196 inhibitor, miR-NC inhibitor, pcDNA-DDX3, pcDNA-3.1, shDDX3 or shCtrl at $37^{\circ} \mathrm{C}$. After $24 \mathrm{~h}$ of incubation, cell proliferation was analyzed.

Apoptosis analysis. Cell apoptosis assays were conducted via flow cytometry using an Annexin V-FITC Apoptosis Detection kit (Beyotime Institute of Biotechnology). Briefly, the OVCAR3 and CAOV-3 cells were seeded into 6-well plates at a density of $1 \times 10^{5}$ cells/well. After transfection, cells were collected using trypsin diluted in PBS and the supernatant was removed. Subsequently, Annexin-V/FITC (5 $\mu \mathrm{l})$ and propidium iodide $(10 \mu \mathrm{l})$ were added to cells, and incubated for $15 \mathrm{~min}$ at room temperature in the dark. The percentage of apoptotic cells was analyzed within $1 \mathrm{~h}$ using a FACSCalibur flow cytometer (BD Biosciences) and BD CellQuestTM software (v5; BD Biosciences). The Q3 data reflected the apoptotic rate in this study.

Luciferase reporter assay. Using the online tool DIANA (v8; DIANA FEA BV), DDX3 was considered to be a putative target gene of miR-196a. For generation of the DDX3 luciferase reporter plasmid, the 3 '-untranslated region (3'-UTR) containing the miR-196a binding site or mutant site was cloned into pmirGLO luciferase vectors (Promega Corporation). Subsequently, $1 \times 10^{6}$ OVCAR3 cells were co-transfected with $2 \square \mathrm{g}$ constructed luciferase vectors accompanied with miR196a mimic or miR-NC mimic $(50 \mathrm{nM})$ by Lipofectamine ${ }^{\circledR}$ 2000 (Invitrogen; Thermo Fisher Scientific, Inc.). Renilla plasmid (hRluc-neo) was used as an internal control. Cells were collected and lysed after $48 \mathrm{~h}$ of transfection. The fluorescence activity was detected using the luciferase reporter assay system (Promega Corporation), according to the manufacturer's instructions. The firefly luciferase activity was normalized to Renilla luciferase activity.

Western blotting. In the present study, western blot analysis was conducted according to the methods detailed below. Total proteins were extracted from OVCAR3 cells using a Protein Isolation kit (Tiangen Biotech Co.,Ltd.), and the protein concentrations were quantified using a Pierce bicinchoninic acid assay (Pierce; Thermo Fisher Scientific, Inc.). Equal amounts of protein samples $(40 \mu \mathrm{g})$ were separated by SDS-PAGE on a $10 \%$ gel before being transferred to a PVDF membrane (EMD Millipore) and blocked with 5\% skimmed milk at $25^{\circ} \mathrm{C}$ for $1 \mathrm{~h}$. After blocking, the proteins were incubated with the following primary antibodies overnight at $4^{\circ} \mathrm{C}$, and then probed with the secondary antibody at $37^{\circ} \mathrm{C}$ for $1 \mathrm{~h}$. The antibodies against DDX3 (1:1,000; cat. no. PA5-17165; Invitrogen; Thermo Fisher Scientific, Inc.), PTEN (1:1,000; cat. no. 9188), phosphorylated (p)-PI3K (1: 1,000; cat. no.17366), total (t)-PI3K (t-PI3K) (1:1,000; cat. no. 4255), p-AKT $(1: 1,000$; cat. no. 4060) and t-AKT (1:1,000; cat. no. 4685) (all obtained from Cell Signaling Technology, Inc.) were used as primary antibodies, whereas peroxidase-conjugated anti-IgG (1:5,000; cat. no. 7074; Cell Signaling Technology, Inc.) was used as a secondary antibody. Monoclonal $\alpha$-tubulin (1:1,000; cat. no. 2125; Cell Signaling Technology, Inc.) and GAPDH antibodies (1:1,000; cat. no. 5174; Cell Signaling Technology, Inc.) were used as controls. Bands were detected using an enhanced chemiluminescent kit (Pierce; Thermo Fisher Scientific, Inc.), and data were semi-quantified using ImageJ software v1.41 (National Institutes of Health).

Statistical analysis. GraphPad Prism 5 software v8.0 (GraphPad Software, Inc.) was used for all statistical analyses. All experiments were carried out in triplicate, and data are presented as the mean \pm SD. A paired Student's t-test was performed to distinguish differences between two groups and one-way ANOVA followed by Tukey's post hoc test were performed for multiple comparisons. $\mathrm{P}<0.05$ was considered to indicate a statistically significant difference.

\section{Results}

miR-196a is upregulated in ovarian cancer cells and tumor tissues. To investigate the relationship between miR-196a and ovarian cancer cells, the expression levels of miR-196a in 30 pairs of epithelial ovarian cancer tissues and corresponding non-tumor tissues were measured using RT-qPCR. It was observed that the expression of miR-196a was significantly higher in ovarian cancer tissues relative to non-tumor tissues (Fig. 1A). Furthermore, the expression of miR-196a was examined in ovarian cancer cell lines (OVCAR3, CAOV-3, A2780 and ES-2) and the immortalized ovarian epithelial cell line HS832. The results were similar to those from the clinical data: The expression of miR-196a was significantly upregulated in ovarian cancer cells (Fig. 1B). Taken together, these findings indicated that miR-196a expression was upregulated in ovarian cancer.

miR-196a promotes cell proliferation and attenuates apoptosis of ovarian cancer cells. To investigate the function of miR-196a in ovarian cancer progression, miR-196a mimic and miR-196a inhibitor were transfected into the ovarian cancer cell lines OVCAR3 and CAOV-3 to induce overexpression or silencing of miR-196a, respectively. OVAR3 and CAOV-3 were chosen for these subsequent experiments, as miR-196a expression was higher in these cell lines than in the other cell lines tested, and both cell lines are the two most commonly used in human ovarian cancer research $(18,19)$. The miR-196a mimic 
A

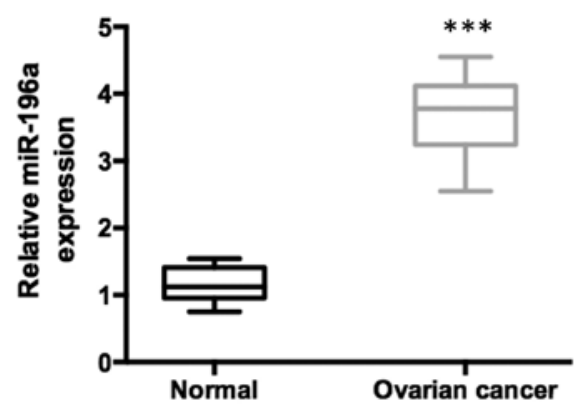

B

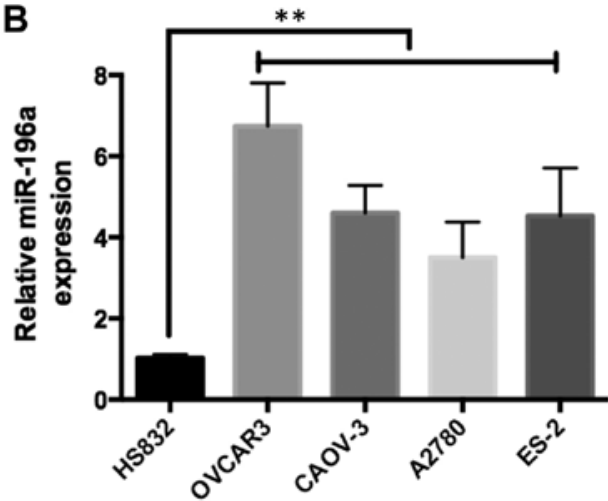

Figure 1. miR-196a expression is significantly increased in tumor tissues of patients with ovarian cancer and ovarian cancer cell lines. (A) Expression levels of miR-196a in 30 pairs of epithelial ovarian cancer tissues and their adjacent, non-cancerous normal ovarian cancer tissues and (B) ovarian cancer cell lines, including OVCAR3, CAOV-3, A2780, ES-2 and immortalized ovarian epithelial cell line HS832 were detected by reverse transcription-quantitative PCR. U6 was used as the internal control. ${ }^{* *} \mathrm{P}<0.01$ and ${ }^{* * *} \mathrm{P}<0.001$. miR, microRNA.

A

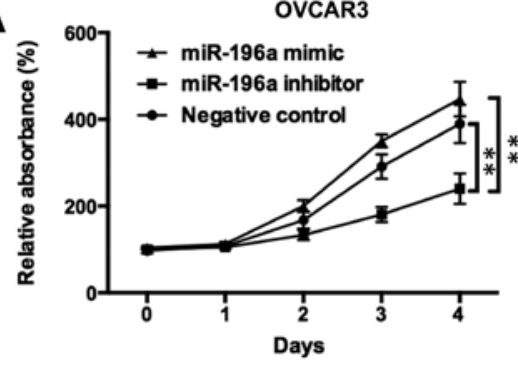

B

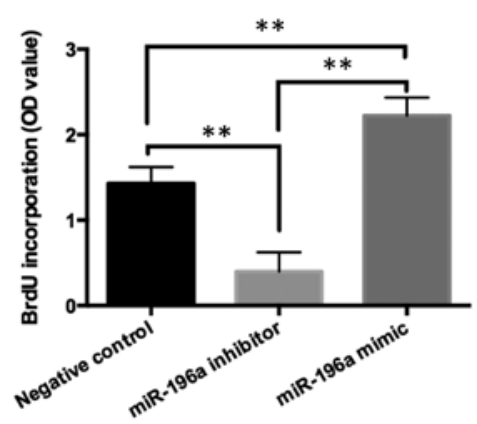

C

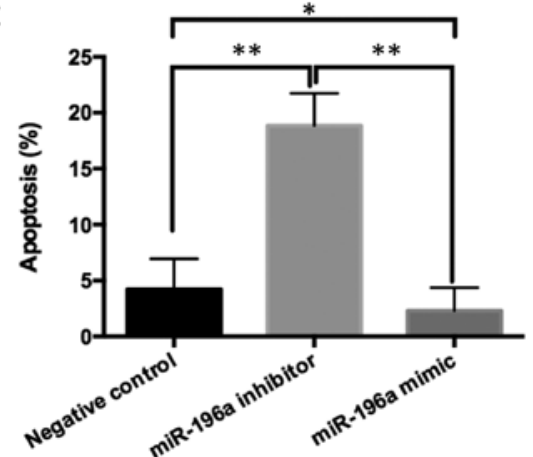

CAOV-3

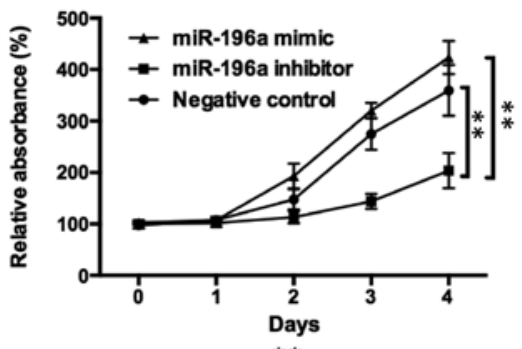

**
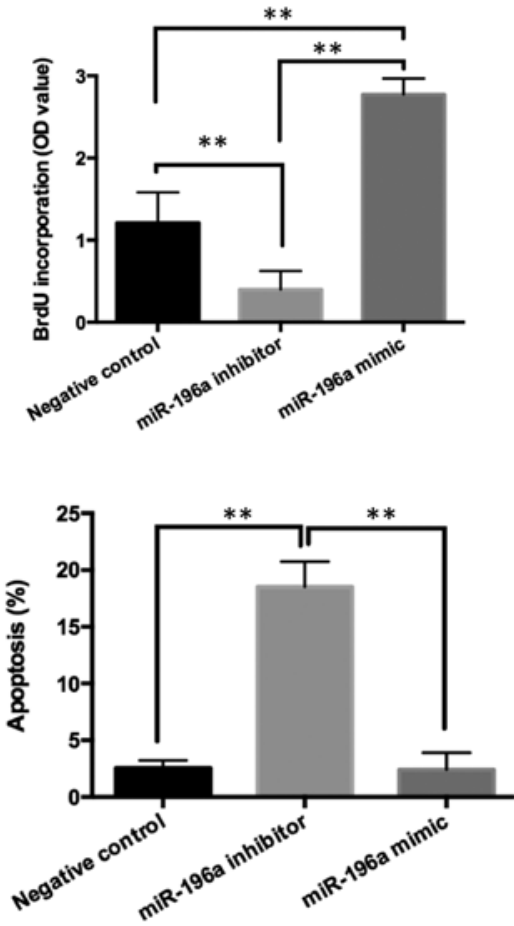

Figure 2. miR-196a promotes proliferation and inhibits apoptosis of ovarian cancer cells. (A) miR-196a inhibitor was transfected into OVCAR3 or CAOV-3 cells and induced a significantly lower proliferation rate than the miR-196a mimic-transfected group and miR-negative control-transfected group as controls. (B) Significantly decreased proliferation levels in OVCAR3 and CAOV-3 cells transfected with miR-221 inhibitors and markedly upregulated cell proliferation of the miR-196a mimic-transfected group compared with that in the controls were observed by ELISA-BrdU assay. (C) miR-196a inhibitor or miR-196a mimic were transfected into OVCAR3 and CAOV-3 cells. miR-196a inhibitor increased the percentage of apoptotic cells compared with controls. ${ }^{*} \mathrm{P}<0.05$ and ${ }^{* *} \mathrm{P}<0.01$. miR, microRNA; OD, optical density.

significantly increased miR-196a expression levels compared with miR-NC mimic both in OVCAR3 and CAOV-3 cell lines, whereas the miR-196a inhibitor significantly decreased
miR-196a expression compared with the miR-NC inhibitor (Fig. S1). As miR-NC mimic and miR-NC inhibitor transfection had no effect on the expression of miR-196a, the control 
A

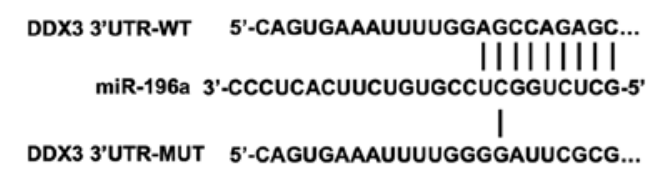

C

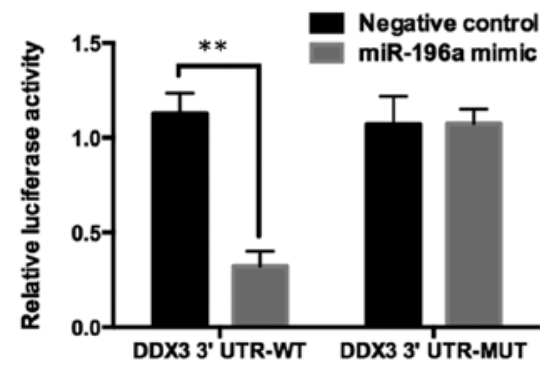

B
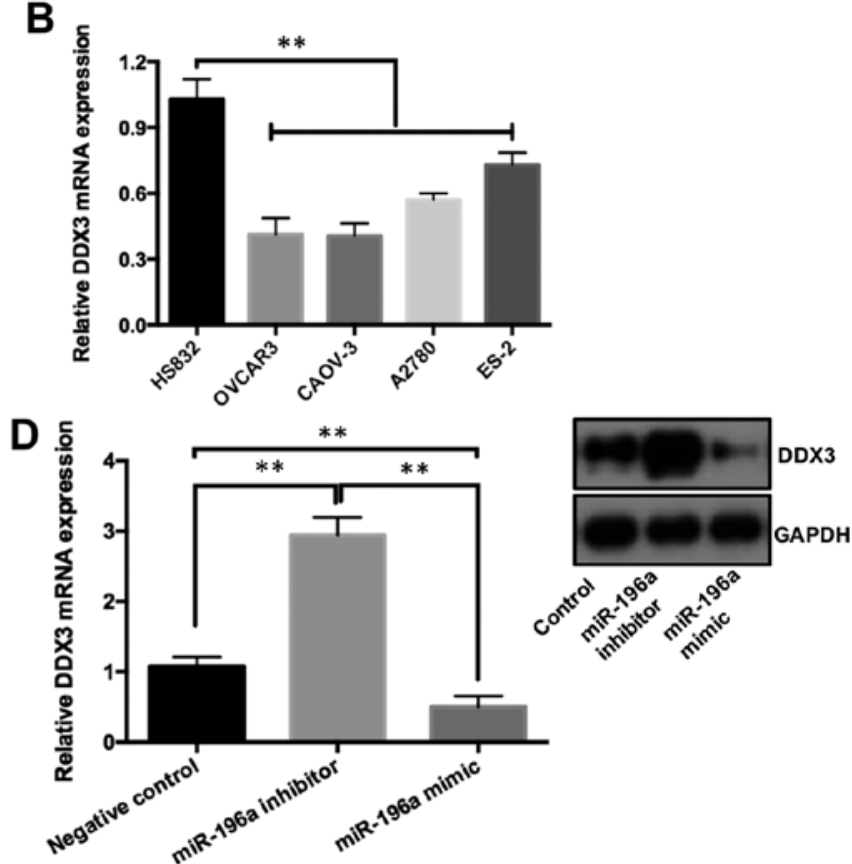

Figure 3. DDX3 is a direct target of miR-196a. (A) Bioinformatics-based target prediction analysis showed that DDX3 is a potential target of miR-196a. (B) mRNA expression levels of DDX3 were markedly decreased in ovarian cell lines compared with in HS832 cells. (C) Luciferase reporter assay showed that in the miR-196a mimic group, a significantly decreased luciferase activity driven by DDX3 was observed compared with that in the negative control and DDX3-MUT group. (D) mRNA and protein expression levels of DDX3 were measured in OVCAR3 cells transfected with the miR-196a mimic or inhibitor. ${ }^{* *} \mathrm{P}<0.01$. miR, microRNA; WT, wild-type; UTR, untranslated region; MUT, mutated; DDX3, DEAD box RNA helicase 3.

A

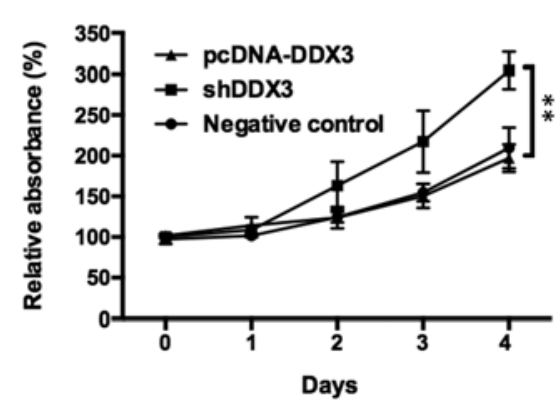

B

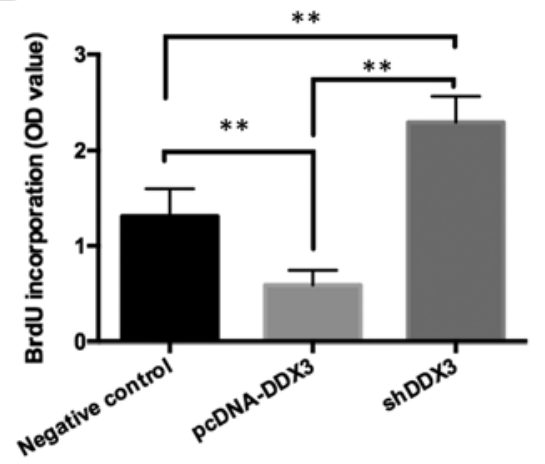

C

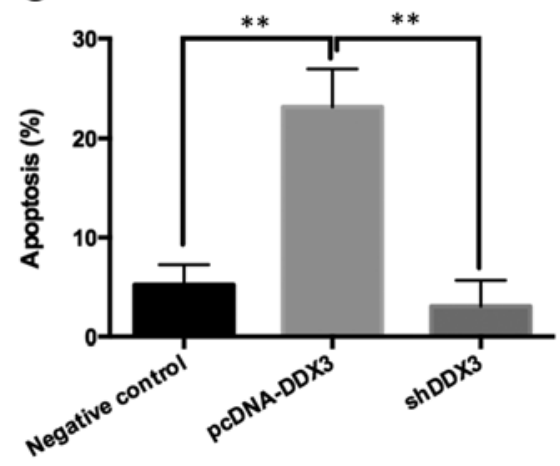

Figure 4. Inhibition of DDX3 promotes cell proliferation and decreases apoptosis of ovarian cancer cells. (A) Decreased cell viability was observed in the pcDNA-DDX3 group compared with the group transfected with shDDX3. (B) Significantly reduced cell proliferation was observed in the pcDNA-DDX3 group compared with the control. (C) Significantly increased the percentage of apoptotic cells was detected in the pcDNA-DDX3 group compared with the control. ${ }^{* *} \mathrm{P}<0.01$. sh, short hairpin; DDX3, DEAD box RNA helicase 3; OD, optical density.

data of the mimic and inhibitor were combined as a $\mathrm{NC}$ for comparative analysis. It was also shown that, compared with the NC, miR-196a mimic promoted cell viability and proliferation (Fig. 2A and B). In contrast, miR-196a inhibitor increased the apoptosis of ovarian cancer cells (Figs. 2C and S2). Collectively, these data suggested that miR-196a may promote cell proliferation and attenuate apoptosis in ovarian cancer.

DDX3 is a direct target of miR-196a. Previous studies have shown that DDX3 mediated miRNA expression, and therefore regulated cell proliferation and metastasis in various cancers $(15,20)$. Using the DIANA tool to uncover potential targets of miR-196a,DDX3 was found to be predicted as a direct target (Fig. 3A). Furthermore, RT-qPCR analysis showed that the expression of DDX3 was downregulated in various ovarian cancer cell lines (Fig. 3B). Luciferase reporter assays were performed to verify the direct interaction between miR-196a and DDX3. It was demonstrated that miR-196a mimic significantly reduced the luciferase activity of the reporter that contained the wild-type DDX3 3'-UTR; in contrast, the luciferase activity of the DDX3 3'-UTR containing a mutation of the miR-196a binding site showed no change in activity (Fig. 3C). In addition, DDX3 expression in cells transfected with miR-196a mimic or inhibitor were examined by RT-qPCR and western blotting. It was shown that DDX3 mRNA expression was significantly reduced by the miR-196a mimic but increased by the miR-196a inhibitor (Fig. 3D). Similar to the RT-qPCR data, the protein expression levels of DDX3 were markedly increased when 
A
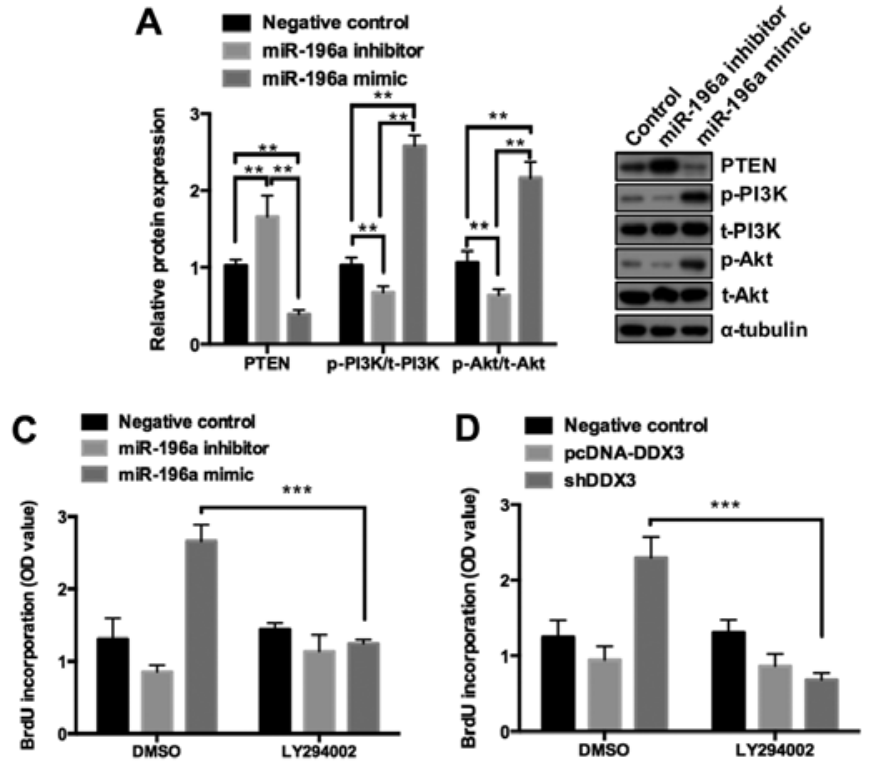
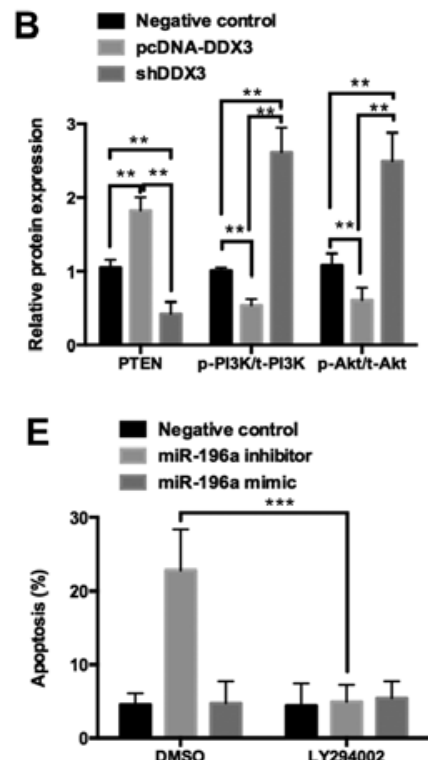
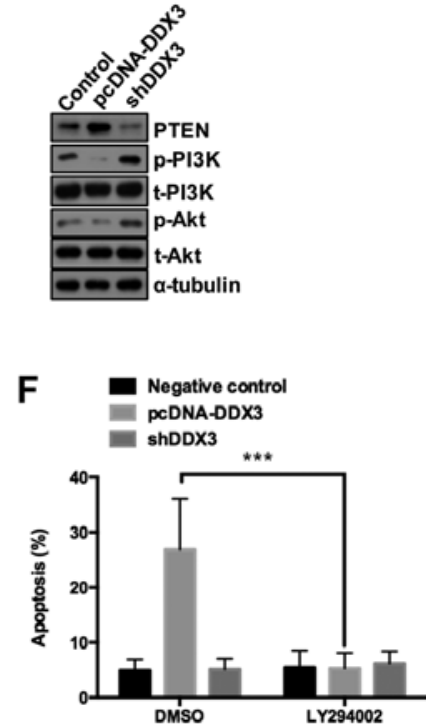

Figure 5. miR-196a promotes cell proliferation by targeting DDX3 via regulating the PTEN/PI3K/AKT signaling pathway. (A and B) Western blot analysis was performed to determine the expression levels of PTEN, p-PI3K, t-PI3K, p-Akt and t-Akt in OVCAR3 cells transfected with miR-196a mimic, miR-196a inhibitor, pcDNA-DDX3, shDDX3 and corresponding control. OVCAR3 cells were pretreated with DMSO or LY294002 for $6 \mathrm{~h}$ and then transfected with miR-196a mimic, miR-196a inhibitor, pcDNA-DDX3, shDDX3 and corresponding control for 24 h. (C and D) Results of the ELISA-BrdU assays performed to analyze cell proliferation and $(\mathrm{E}$ and $\mathrm{F})$ results of the flow cytometry used to evaluate the percentage of apoptotic cells. ${ }^{* *} \mathrm{P}<0.01$ and ${ }^{* * *} \mathrm{P}<0.001$. miR, microRNA; p, phosphorylated; t, total; sh, short hairpin; DDX3, DEAD box RNA helicase 3; OD, optical density.

the miR-196a inhibitor was transfected, whereas they were decreased when miR-196a was overexpressed (Fig. 3D).

Inhibition of DDX3 promotes cell proliferation and decreases apoptosis of ovarian cancer cells. To confirm that miR-196a regulated ovarian cancer cell proliferation and apoptosis by targeting DDX3, OVCAR3 cells were transfected with pcDNA-DDX3, shDDX3, or control pcDNA3.1 vector and shCtrl into and analyzed using MTT, cell proliferation and flow cytometry assays. pcDNA-DDX3 was transfected into OVCAR3 cells to significantly upregulate DDX3 expression compared with the control, whereas shDDX3 was transfected into cells to markedly downregulate DDX3 expression (Fig. S3). As pcDNA3.1 and shCtrl transfection had no effect on the expression of DDX3, the control data of the pcDNA vector and shCtrl were combined as a NC for comparative analysis. Increased cell viability and proliferation, as well as reduced apoptosis, were observed in the shDDX3 group relative to the pcDNA-DDX3 or control groups (Figs. 4A-C and S4). In summary, these results indicated that miR-196a may promote cell proliferation and attenuate apoptosis in ovarian cancer through the targeting of DDX3.

DDX3 reverses the effects of miR-196a on ovarian cancer cells via the PTEN/PI3K/AKT signaling pathway. To investigate the molecular mechanism underlying miR-196a targeting of DDX3 in ovarian cancer, miR-196a mimic, miR-196a inhibitor, pcDNA-DDX3, shDDX3 and a corresponding control were transfected into OVCAR3 ovarian cancer cells to assess the effects on the PTEN/PI3K/AKT pathway. As shown in Fig. 5A, the miR-196a mimic decreased protein expression of PTEN, while also inducing PI3K and AKT phosphorylation. Previous studies have reported that inhibition of PTEN as a tumor suppressor may activate the expression of PI3K and AKT, thereby increasing survival of various tumors $(21,22)$. Notably, in the present study, downregulation of DDX3 attenuated the expression of PTEN and therefore increased the expression of p-PI3K and p-AKT (Fig. 5B); thus, DDX3 may play a role in regulating ovarian cancer cell proliferation. Furthermore, the effect of PTEN/PI3K/AKT pathway inhibition on cell proliferation and apoptosis was explored. It was revealed that the miR-196a mimic or shDDX3-mediated upregulation of cell proliferation and miR-196a inhibitor or pcDNA-DDX3-mediated inhibition of apoptosis were blocked by pretreatment with LY294002, which is an AKT inhibitor (Figs. 5C-F and S5). Taken together, these results suggested that miR-196a may promote ovarian cancer cell proliferation and attenuate apoptosis by targeting DDX3 through PTEN/PI3K/AKT signaling pathway regulation.

\section{Discussion}

miR-196a is highly expressed in ovarian cancer and is associated with various other types of cancer; therefore, it could represent an important biomarker for cancer diagnosis and prognosis $(12,13)$. In epithelial ovarian cancer progression, Yang et al (14) found that miR-196a promoted ovarian cancer cell migration and invasion through the downstream target gene HOXA10; however, these authors did not report the regulatory mechanisms of miR-196a and HOXA10. Notably, there are few studies on the precise regulatory mechanisms of miR-196a in ovarian cancer progression. Therefore, in the present study, the relationship between miR-196a and ovarian cancer was investigated in detail. It was found that the expression of miR-196a was increased in ovarian cancer tissues and cell lines, and that miR-196a promoted ovarian cell 
proliferation and suppressed cell apoptosis. In addition, transfection of miR-196a mimics reduced DDX3 expression levels in cells and miR-196a bound to the 3'-UTR of DDX3. Thus, DDX3 was demonstrated to be a direct target of miR-196a, and importantly, attenuated the effect of miR-196a on ovarian cancer. After further mechanistic studies, it was revealed that miR-196a promoted cell viability and proliferation but attenuated cell apoptosis by inhibiting DDX3 via PTEN/PI3K/AKT pathway regulation. To the best of our knowledge, the present study is the first to demonstrate an association between miR-196a and DDX3 in ovarian cancer, and has provided a novel insight into the molecular mechanisms of ovarian cancer progression.

As previously reported, the DDX3 gene is similar to a 'double-edged sword': Depending on the type of cancer, it either contributes to cancer progression or functions as a tumor suppressor (23). For example, DDX3 exhibited oncogenic functions in breast cancer, in which overexpression of the gene promoted cell growth and proliferation in breast epithelial cancer cells $(16,24)$. However, in colorectal cancer, the knockdown of DDX3 promoted colon cancer progression via the Snail/E-cadherin pathway (15). Although the mechanism of this dual role of DDX3 in various types of cancer has not been fully elucidated, the results of the present study suggested that the regulatory function of miRNAs may have an important role in these processes. In previous studies, several DDX genes, including DDX1 and DDX10, have been reported to play tumor-suppressing roles in ovarian cancer progression $(25,26)$. In addition, the present study demonstrated a downregulation of DDX3 in ovarian cancer cell lines. Furthermore, overexpression of DDX3 decreased cell proliferation and induced apoptosis, which suggested that, similar to other DDX genes, DDX3 may act as an ovarian cancer tumor suppressor.

The present study indicated that miR-196a targeted DDX3 to promote cell proliferation through the PTEN/PI3K/AKT signaling pathway. PTEN, a well-established tumor suppressor, is known to contribute to cancer development and progression (27). In addition, the PTEN/PI3K/AKT signaling pathway has a confirmed link to the carcinogenesis of various tumors, including hepatocellular carcinoma, gastric cancer and pancreatic cancer $(28,29)$. PTEN has been reported to attenuate cancer cell viability and growth by suppressing the PI3K/AKT signaling pathway, while downregulation of PTEN expression may result in the activation of AKT, and the promotion of cell proliferation and angiogenesis (30). Collectively, the results of the present study supported the hypothesis that overexpression of miR-196a promoted ovarian cell growth and proliferation via downregulation of PTEN expression. These findings suggested that miR-196a may be a negative regulator of the PTEN/PI3K/AKT signaling pathway. Moreover, it was suggested that overexpression of DDX3 reversed miR-196a-mediated tumor progression by activating PTEN expression and suppressing AKT activity, thereby blocking the miR-196a-mediated effects of the PTEN/PI3K/AKT signaling pathway.

In summary, the present study provides a new insight into the regulatory mechanisms that underlie the effects of miR-196a on ovarian cancer progression. To the best of our knowledge, the present study is the first to report an association between miR-196a and DDX3, and has demonstrated that
miR-196a may promote cell proliferation and inhibit apoptosis in ovarian cancer through attenuation of DDX3 expression via PTEN/PI3K/AKT signaling pathway regulation. It is therefore suggested that miR-196a and DDX3 are potential targets for the development of future ovarian cancer treatments.

\section{Acknowledgements}

Not applicable.

\section{Funding}

No funding was received.

\section{Availability of data and materials}

The datasets used and/or analyzed during the current study are available from the corresponding author on reasonable request.

\section{Authors' contributions}

JN and WZ conceived and designed the experiments; JN, LC, LL, MW and QR performed the experiments and analyzed the data; JN, LC and WZ wrote the manuscript. All authors read and approved the final manuscript.

\section{Ethics approval and consent to participate}

Written informed consent was obtained from all patients, and the present study was approved by the Institutional Ethics Committee and Academic Committee of The Second Affiliated Hospital of Soochow University.

\section{Patient consent for publication}

Not applicable.

\section{Competing interests}

The authors declare that they have no competing interests.

\section{References}

1. Webb PM and Jordan SJ: Epidemiology of epithelial ovarian cancer. Best Pract Res Clin Obstet Gynaecol 41: 3-14, 2017.

2. Fathalla MF: Incessant ovulation--a factor in ovarian neoplasia? Lancet 2: 163, 1971.

3. Cramer DW and Welch WR: Determinants of ovarian cancer risk. II. Inferences regarding pathogenesis. J Natl Cancer Inst 71: 717-721, 1983.

4. Orr B and Edwards RP: Diagnosis and treatment of ovarian cancer. Hematol Oncol Clin North Am 32: 943-964, 2018.

5. Lu TX, Rothenberg ME and Micro RNA: MicroRNA. J Allergy Clin Immunol 141: 1202-1207, 2018

6. Rupaimoole R and Slack FJ: MicroRNA therapeutics: Towards a new era for the management of cancer and other diseases. Nat Rev Drug Discov 16: 203-222, 2017.

7. Thorsen SB, Obad S, Jensen NF, Stenvang J and Kauppinen S: The therapeutic potential of microRNAs in cancer. Cancer J 18: 275-284, 2012

8. Nana-Sinkam SP and Croce CM: Clinical applications for microRNAs in cancer. Clin Pharmacol Ther 93: 98-104, 2013.

9. Tutar L, Tutar E, Özgür A and Tutar Y: Therapeutic Targeting of microRNAs in Cancer: Future Perspectives. Drug Dev Res 76: 382-388, 2015. 
10. Zhong LN, Zhang YZ, Li H, Fu HL, Lv CX and Jia XJ: Overexpressed miR-196a accelerates osteogenic differentiation in osteoporotic mice via GNAS-dependent Hedgehog signaling pathway. J Cell Biochem 120: 19422-19431, 2019.

11. RenW,Wu S, Wu Y,Liu T,ZhaoX and Li Y:MicroRNA-196a/-196b regulate the progression of hepatocellular carcinoma through modulating the JAK/STAT pathway via targeting SOCS2. Cel Death Dis 10: 333, 2019.

12. Fan Y, Fan J, Huang L, Ye M, Huang Z, Wang Y, Li Q and Huang J: Increased expression of microRNA-196a predicts poor prognosis in human ovarian carcinoma. Int J Clin Exp Pathol 8: 4132-4137, 2015

13. Lukács J, Soltész B, Penyige A, Nagy B and Póka R: Identification of miR-146a and miR-196a-2 single nucleotide polymorphisms at patients with high-grade serous ovarian cancer. J Biotechnol 297: 54-57, 2019.

14. Yang B, Li SZ, Ma L, Liu HL, Liu J and Shao JJ: Expression and mechanism of action of miR-196a in epithelial ovarian cancer. Asian Pac J Trop Med 9: 1105-1110, 2016.

15. Su CY, Lin TC, Lin YF, Chen MH, Lee CH, Wang HY, Lee YC, Liu YP, Chen CL and Hsiao M: DDX3 as a strongest prognosis marker and its downregulation promotes metastasis in colorectal cancer. Oncotarget 6: 18602-18612, 2015.

16. Botlagunta M, Vesuna F, Mironchik Y, Raman A, Lisok A, Winnard P Jr, Mukadam S, Van Diest P, Chen JH, Farabaugh P, et al: Oncogenic role of DDX3 in breast cancer biogenesis. Oncogene 27: 3912-3922, 2008

17. Livak KJ and Schmittgen TD: Analysis of relative gene expression data using real-time quantitative PCR and the 2(-Delta Delta C(T)) Method. Methods 25: 402-408, 2001.

18. Blomquist $\mathrm{CH}$, Leung BS, Zhang R, Zhu Y and Chang PM: Properties and regulation of $17 \beta$-hydroxysteroid oxidoreductase of OVCAR-3, CAOV-3, and A431 cells: Effects of epidermal growth factor, estradiol, and progesterone. J Cell Biochem 59: 409-417, 1995.

19. CheungLWT,LeungPCK and Wong AST:Gonadotropin-releasing hormone promotes ovarian cancer cell invasiveness through c-Jun NH2-terminal kinase-mediated activation of matrix metalloproteinase (MMP)-2 and MMP-9. Cancer Res 66: 10902-10910, 2006.

20. You S, Wang F, Hu Q, Li P, Zhang C, Yu Y, Zhang Y, Li Q, Bao Q, Liu P, et al: Abnormal expression of YEATS4 associates with poor prognosis and promotes cell proliferation of hepatic carcinoma cell by regulation the TCEA1/DDX3 axis. Am J Cancer Res 8: 2076-2087, 2018.
21. Kim MJ, Lee SJ, Ryu JH, Kim SH, Kwon IC and Roberts TM: Combination of KRAS gene silencing and PI3K inhibition for ovarian cancer treatment. J Control Release 318: 98-108, 2020.

22. Patch AM, Christie EL, Etemadmoghadam D, Garsed DW, George J, Fereday S, Nones K, Cowin P, Alsop K, Bailey PJ, et al; Australian Ovarian Cancer Study Group: Whole-genome characterization of chemoresistant ovarian cancer. Nature 521: 489-494, 2015.

23. He Y, Zhang D, Yang Y, Wang X, Zhao X, Zhang P, Zhu H, Xu N and Liang S: A double-edged function of DDX3, as an oncogene or tumor suppressor, in cancer progression (Review). Oncol Rep 39: 883-892, 2018. (Review).

24. Heerma van Voss MR, Schrijver WA, TerHoeve ND, HoefnagelLD, Manson QF, van der Wall E, Raman V and van Diest PJ; Dutch Distant Breast Cancer Metastases Consortium: The prognostic effect of DDX3 upregulation in distant breast cancer metastases. Clin Exp Metastasis 34: 85-92, 2017.

25. Gai M, Bo Q and Qi L: Epigenetic down-regulated DDX10 promotes cell proliferation through $\mathrm{Akt} / \mathrm{NF}-x \mathrm{~B}$ pathway in ovarian cancer. Biochem Biophys Res Commun 469: 1000-1005, 2016.

26. Han C, Liu Y, Wan G, Choi HJ, Zhao L, Ivan C, He X, Sood AK, Zhang $X$ and Lu X: The RNA-binding protein DDX1 promotes primary microRNA maturation and inhibits ovarian tumor progression. Cell Rep 8: 1447-1460, 2014.

27. Carnero A and Paramio JM: The PTEN/PI3K/AKT Pathway in vivo, Cancer Mouse Models. Front Oncol 4: 252, 2014.

28. Wise HM, Hermida MA and Leslie NR: Prostate cancer, PI3K, PTEN and prognosis. Clin Sci (Lond) 131: 197-210, 2017.

29. Nakahata S, Ichikawa T, Maneesaay P, Saito Y, Nagai K, Tamura T, Manachai N, Yamakawa N, Hamasaki M, Kitabayashi I, et al: Loss of NDRG2 expression activates PI3K-AKT signalling via PTEN phosphorylation in ATLL and other cancers. Nat Commun 5: 3393, 2014

30. Xia M, Tong JH, Ji NN, Duan ML, Tan YH and Xu JG: Tramadol regulates proliferation, migration and invasion via PTEN/PI3K/AKT signaling in lung adenocarcinoma cells. Eur Rev Med Pharmacol Sci 20: 2573-2580, 2016.

This work is licensed under a Creative Commons Attribution-NonCommercial-NoDerivatives 4.0 International (CC BY-NC-ND 4.0) License. 